\title{
Cells containing factor XIIIa and pulmonary fibrosis induced by bleomycin
}

\author{
M Toida, Y Okumura, T Takami
}

Department of Oral

Surgery and Oral

Medicine, and

Pathology Division,

Clinical Laboratory,

Ibi General Hospital,

2547-4 Mina,

Ibigawa-cho, Ibi-gun,

Gifu-prefecture,

501-06, Japan

M Toida

Department of Oral and Maxillo-Facial

Surgery, Gifu

University School of

Medicine

Y Okumura

Department of

Pathology, Gifu

University School of

Medicine

T Takami

Correspondence to:

Dr M Toida

Accepted for publication

5 September 1990

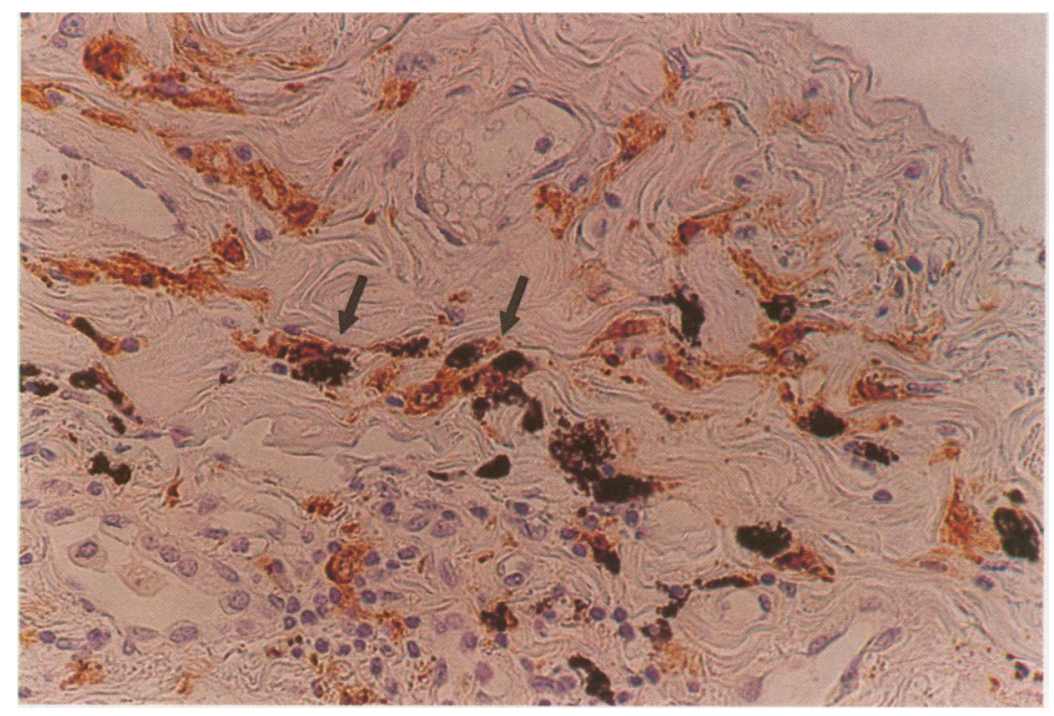

Figure 1 Numerous cells containing FXIIIa are seen in the subpleural fibrous connective tissue. Note the coexistence of both dust particles (black) and FXIIIa positive reaction products (brown) in some interstitial cells (arrows).

(Immunoperoxidase staining for FXIIIa). by many other agents affecting the lung. ${ }^{1}$ The histological features include intra-alveolar accumulation of proteinaceous for fibrinous exudate, proliferation of alveolar cells, interstitial and intra-alveolar fibrosis, and squamous metaplasia with epithelial dysplasia of the distal air spaces. ${ }^{1}$

FXIIIa has been detected immunohistochemically in certain interstitial cells of various human tissues, ${ }^{23}$ though the distribution of FXIIIa was not examined in pulmonary tissues. More recently, it has been suggested that these cells have an important role in the process of fibrosis in various lesions. ${ }^{45}$

\section{Methods}

Fifty five specimens of lung tissue, consisting of 24 normal and 31 pulmonary fibrotic tissues were obtained from five necropsies. The tissues were almost intact in two of the cases (a 40 year old man and a 54 year old woman), and the tissues from the other three cases (a 61 year old woman, a 69 year old man, and a 79 year old man) showed pulmonary fibrosis. Specimens about $5 \mathrm{~mm}$ thick were cut from the excised lung and immediately fixed in glacial acetic acid $1 \%(\mathrm{v} / \mathrm{v})$ in $95 \%$ ethanol at $4^{\circ} \mathrm{C}$ for 12 to 24 hours. The specimens were embedded in paraffin wax and cut into $4 \mu \mathrm{m}$ thick sections. Each section was dewaxed, hydrated, and stained with haematoxylin and eosin and azocarmine and aniline blue (AZAN).

Collagenous components were stained using the Sirius red F3BA method and a collagen stain kit (Collagen Gijutsu Kenshu-Kai, Tokyo, Japan).

Immunoperoxidase staining for FXIIIa was performed by means of the avidin-biotinperoxidase complex (ABC) method with rabbit antiseum against human FXIIIa (Behringwerke AG, Marburg, Germany) diluted 1 in 400 and the rabbit IgG Vectastain ABC kit (Vector Laboratories, Burlingame, California, USA). The specific antigenantibody reaction product was visualised by 3,3'-diaminobenzidine tetrahydrochloride and hydrogen peroxide. Counterstaining was performed with Mayer's haematoxylin. For control slides, normal rabbit serum at the same dilution was used instead of anti-FXIIIa antiserum.

Details on both Sirius red F3BA collagen staining and immunoperoxidase staining procedures have already been described. ${ }^{4}$ 


\section{Results}

In normal tissues FXIIIa was detected in a few interstitial cells. They were mainly spheroidal or spindle-shaped. Alveolar macrophages also exhibited a weakly positive reaction to FXIIIa. Alveolar epithelium, pleural mesothelium, vascular endothelium and bronchial glandular cells were all negative for FXIIIa.

In fibrotic tissues numerous cell containing FXIIIa were observed, especially in the subpleural area (fig 1) and around the blood vessels of alveolar septa, where slight to moderate fibrosis was seen. These cells were mainly dendritic in shape, and some of them were dust-laden (fig 1). In the alveolar spaces, where proteinaceous or fibrinous exudation and intraalveolar fibrosis were prominent, the alveolar macrophages exhibited a strong positive reaction for FXIIIa (fig 2). In the highly collagenous scar-like areas the interstitial cells containing FXIIIa were fewer in number. They were mainly slender, spindleshaped, and exhibited a weak positive reaction for FXIIIa.

\section{Discussion}

Recently, it has been shown that FXIIIa can catalyse in vitro not only the crosslinking of fibrin monomers to each other, but also the crosslinking of fibronectin monomers to each other $^{6}$ and to type I and type III collagens. This may be important not only for the stabilisation of fibrin clots, but also during the process of fibrosis. Recent immunohistochemical studies have also indicated findings suggesting that the cells containing FXIIIa

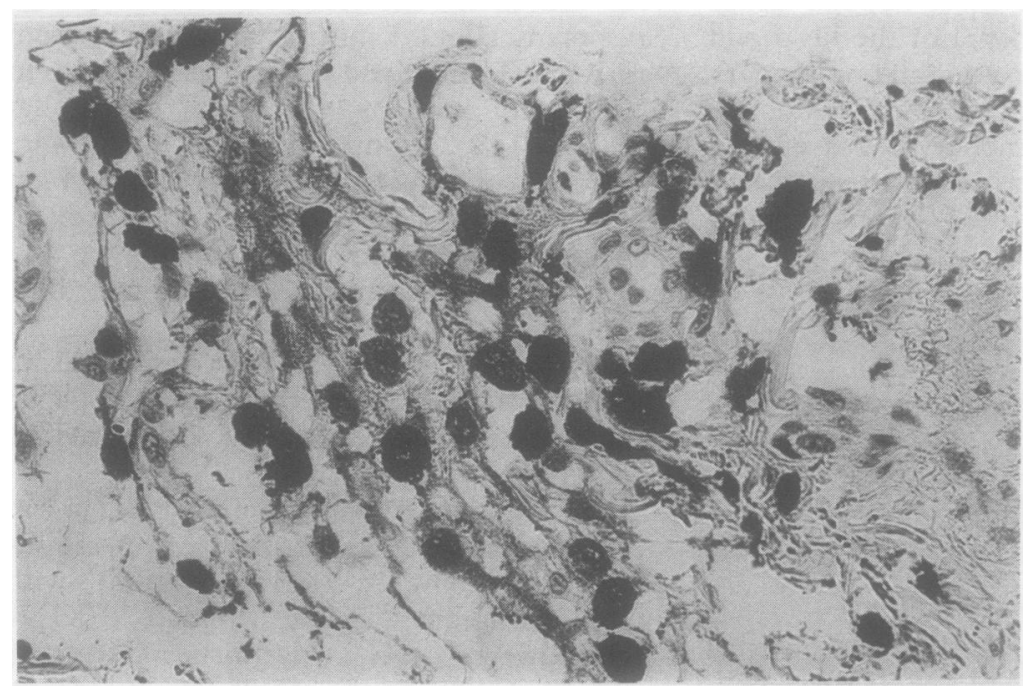

Figure 2 Numerous macrophages showing an intense positive reaction for FXIIIa are found in the alveolar space, in which fibrinous exudate is prominent. (Immunoperoxidase staining for FXIIIa). have an important role during the process of fibrosis in various lesions. ${ }^{45}$ Our study shows a close relation between the distribution of cells containing FXIIIa and that of collagenous components. It has been suggested that alveolar macrophages have an important role in the development of pulmonary fibrosis by releasing various fibroblast proliferation factors, such as fibronectin, ${ }^{8}$ certain types of platelet derived growth factor ${ }^{9}$ and interleukin-1. ${ }^{10}$ Apart from these factors, FXIIIa contained in alveolar macrophages as well as in pulmonary interstitial cells may also have an important role in the development of this lesion. On the basis of the findings obtained in this study we speculate that these cells proliferate before fibrosis develops and that the FXIIIa stimulates fibrosis in the early stage of this lesion, and furthermore, that this role of the cells is diminished as the lesion develops to the final stage.

In this study some of the cells containing FXIIIa were identified morphologically as alveolar macrophages. The dust-laden connective tissue cells containing FXIIIa are also interpreted as being of the macrophage type. There is, however, some doubt as to whether all interstitial cells that contain FXIIIa belong to the macrophage type; and also whether macrophages can in fact act as facultative fibroblasts.

We thank Dr Akitsugu Ojima, the Department of Pathology, Gifu University School of Medicine, for his advice; D Nobumitsu Oka, and Norichika Tatematsu, of the Department of Oral and Maxillo-Facial Surgery, Gifu University School of of Oral and Maxillo-Facial Surgery, Gifu University School of Medicine; and
for their help.

1 Luna WA, Bedrossian WM, Lichtiger B, Salem PA. Interstitial pneumonitis associated with bleomycin therapy. Am J Clin Pathol 1972;58:501-10.

2 Fear JD, Jackson P, Gray C, Miloszewski JKA, Losowsky MS. Localization of factor XIII in human tissues using an immunoperoxidase technique. J Clin Pathol 1984;37: $560-3$.

3 Ádány R, Glukhova MA, Kabakov AY, Muszbek L. Characterization of connective tissue cells containing factor XIII subunit a. J Clin Pathol 1988;41:49-56.

4 Toida M, Watanabe F, Tsai CS, Okutomi T, Tatematsu N, Oka N. Factor XIIIa-containing cells and fibrosis in oral and maxillofacial lesions: An immunohistochemical study. Oral Surg Oral Med Oral Pathol 1989;68:293-9.

5 Penneys NS. Factor XIII expression in the skin: Observations and a hypothesis. J Am Acad Dermatol 1990;22: 484-8.

6 Mosher DF Cross-linking of cold insoluble globulin by fibrin-stabilizing factor. J Biol Chem 1976;250:6614-21. fibrin-stabilizing factor. J Biol Chem 1976;250:6614-21.
Mosher DF. Cross-linking of fibronectin to collagenous Mosher DF. Cross-linking of fibronectin
proteins. Mol Cell Biochem 1984;53:63-8.

proteins. Mol Cell Biochem 1984;53:63-8.
Rennard SI, Hunninghake GW, Bitterman PB, Crystal RG Rennard SI, Hunninghake GW, Bitterman PB, Crystal RG
Production of fibronectin by the human alveolar macrophage: Mechanism for the recruitment of fibroblasts to sites of tissue injury in interstitial lung diseases. Proc Natl Acad Sci USA 1981;78:7147-51.

9 Shimokado K, Raines EW, Madtes DK, Barrett TB, Benditt EP, Ross R. A significant part of macrophage-derived growth factor consists of at least two forms of PDGF. Cell 1985;43:277-86.

10 Suwabe A, Takahashi K, Yasui S, Arai S, Sendo F. Bleomycin-stimulated hamster alveolar macrophages release interleukin-1. Am J Pathol 1988;132:512-20. 